\title{
Composición proximal en algunas especies de pescado y mariscos disponibles en el pacífico costarricense
}

\section{Proximate Composition in Some Species of Fish and Seafood Available on the Costa Rican Pacific Ocean}

\author{
Cristian Fonseca-Rodríguez \\ cristian.fonseca.rodriguez@una.cr \\ Estación de Biología Marina, Escuela de Ciencias Biológicas \\ Universidad Nacional \\ Puntarenas, Costa Rica \\ Fabián Chavarría-Solera \\ fabian.chavarria.solera@una.cr \\ Programa UNA-Campus Sostenible, Universidad Nacional \\ Heredia, Costa Rica
}

Recibido-Received: 10/dic/2015 / Corregido-Corrected: 7/jun /2016.

Aceptado-Accepted: 20/ago/2016 / Publicado-Published: 31/ene /2017.

\begin{abstract}
Resumen
El pescado y los mariscos son alimentos de los más completos por su calidad y cantidad de nutrientes; sin embargo, poco se sabe sobre la composición química de los recursos pesqueros del pacífico costarricense. Por tal razón, el objetivo del presente trabajo es determinar el potencial nutricional de las partes comestibles (fresco) de seis especies de pescados y mariscos de consumo e importancia comercial en la zona pacífica de Costa Rica. Los análisis de la composición proximal: humedad, cenizas, extracto etéreo y proteínas se determinaron según la metodología de AOAC. Los resultados indicaron que el contenido de humedad varió entre 77,13 \% en barracuda (Sphyraena ensis) y 83,12\% en calamar (Lolliguncula panamensis), la ceniza entre $0,84 \%$ en calamar y $1,65 \%$ en almeja de agua dulce (Anodonta luteola), el extracto etéreo entre $1,16 \%$ para el calamar y $2,24 \%$ para la almeja de agua dulce. La almeja de agua dulce presentó el menor porcentaje de proteínas (10,73\%), mientras que el lenguado (Cyclopsetla querna) el mayor porcentaje (19,86\%). Los resultados permiten concluir que las especies estudiadas son una buena alternativa para el consumo humano, por su bajo contenido en grasas y por el aporte proteico.
\end{abstract}

Palabras claves: Pescado, composición proximal, valor nutritivo, análisis químicos, Costa Rica.

\begin{abstract}
Fish and shellfish are the most complete foods due to their quality and quantity of nutrients. However, little is known about the chemical composition of the fishery resources of the Costa Rican Pacific Ocean. Therefore, this study aims to determine the nutritional potential of the (fresh) edible parts of six seafood species for consumption and with commercial importance in the Pacific area of Costa Rica. The proximate composition analysis-moisture, ash, ether extract and protein-were determined by AOAC methodology. The results indicated that the moisture content ranged from $77.13 \%$ for barracuda (Sphyraena ensis) and $83.12 \%$ for squid (Lolliguncula panamensis); ash was between $0.84 \%$ for squid and $1.65 \%$ for freshwater mussel (Anodonta luteola); the ether extract, from
\end{abstract}

Cristian Fonseca-Rodríguez y Fabián Chavarría-Solera

Artículo protegido por licencia Creative Commons: BY-NC-ND / Protected by Creative Commons: BY-NC-ND

Uniciencia es una revista de acceso abierto/ Uniciencia is an Open Access Journal. 
UNICIENCIA Vol. 31, No. 1, pp. 23-28. Enero-junio, 2017.

ISSN Electrónico: 2215-3470

URL: www.revistas.una.ac.cr/uniciencia

DOI: $\underline{\text { http://dx.doi.org/10.15359/ru.31-1.3 }}$

Email: revistauniciencia@una.cr

$1.16 \%$ for squid and $2.24 \%$ for freshwater clam. The freshwater clam had the lowest percentage of protein (10.73\%); while the sole (Cyclopsetla querna) showed the highest percentage (19.86\%). The studied species are a good alternative for human consumption due to their low fat and protein intake.

Keywords: fish, proximate composition, nutritional value, chemical analysis, Costa Rica.

A lo largo del mundo ha sido ampliamente reconocido que los productos de la pesca y de la acuicultura constituyen recursos alimenticios importantes para la mayoría de las sociedades, por su valioso aporte de nutrientes en la dieta humana (Chukwu y Mohammed, 2009) y se les considera como uno de los alimentos más completos por la calidad y cantidad de nutrimentos que aporta (Izquierdo et al., 2000). Se sabe que constituyen una fuente alta en proteína, minerales y vitaminas esenciales de valioso valor biológico (Astorga et al., 2007; Fuentes et al., 2009; Özden 2010a). Más aún, por su bajo contenido en grasas y calidad de ácidos grasos, muchas investigaciones reportan los efectos benéficos del consumo de estos organismos para una buena salud (Castro-González et al., 2007; Perea et al., 2008; Özden 2010b).

La carne de pescado contiene como nutrientes básicos agua, proteínas, grasas, carbohidratos, ceniza y otros nutrientes importantes y sustancias como minerales y vitaminas (Stansby, 1962). Según la FAO (1991), los peces contienen $72 \%$ de agua, $19 \%$ de proteínas y $8 \%$ de grasa. Sin embargo, su composición y, por tanto, su valor nutritivo están influenciados por diversas variables como la especie, edad, medio en que viven, tipo de alimentación, época de captura, entre otros (Nazrul y Razzaq, 2005).

El conocimiento del aporte nutricional de las especies marinas y dulceacuícolas ha sido motivo de extensos estudios en varios países, lo cual ha permitido determinar el valor alimenticio de estos recursos en Latinoamérica (Badillo-Zapata et al., 2010; Cabrera et al., 2005; CastroGonzález et al., 1998; Izquierdo et al., 2000; Izquierdo et al., 2001; López et al., 2006; Romero et al., 2000) como también en otras latitudes (Cox y Hartman, 2005; Fawole et al., 2007; Nurnadia et al., 2011). No obstante, la información acerca de los valores nutricionales y composición química de las especies que se consumen en Costa Rica es escasa y se resume en los trabajos presentados por Báez (1985); Blanco y Montero (1992); Cruz y Villalobos (1993); liménez-Arce (1993); Ruiz et al. (1998); Fonseca et al., (2011); Cruz et al. (2012) y Fonseca et al. (2013).

Por el contrario, la información relativa a la composición proximal (valor nutricional) ha ido evolucionando y cobrando cada vez más importancia para el consumidor sensibilizado con mantener una dieta sana y variada, así como abarcar todas sus necesidades nutritivas. Este hecho, unido a la información escasa y heterogénea de este tipo de alimentos, llevó a la necesidad de realizar esta investigación cuyo objetivo fue brindar información sobre la composición proximal de algunas especies de pescado y mariscos de consumo e importancia comercial en la zona pacífica de Costa Rica.

\section{Metodología}

\section{Recolección y preparación de las muestras}

Se analizó la porción comestible (filetes sin espinas) de 3 especies de pescado y 1 especie de calamar (manto y tentáculos), ambos de consumo cotidiano en Costa Rica. Además, se adquirieron muestras de un langostino extraído con fines de exportación y 1 especie de almeja de agua dulce. En la Tabla 1 se presenta el nombre científico de cada especie; así como los nombres comunes con que se conoce. 
ISSN Electrónico: 2215-3470

DOI: http://dx.doi.org/10.15359/ru.31-1.3
UNICIENCIA Vol. 31, No. 1, pp. 23-28. Enero-junio, 2017.

URL: www.revistas.una.ac.cr/uniciencia Email: revistauniciencia@una.cr

Tabla 1

Nombres científicos y comunes de 6 especies de pescado y mariscos evaluadas para la caracterización de la composición proximal

\begin{tabular}{lll}
\hline Nombre científico & Nombre común en Costa Rica & Nombre común en ingles \\
\hline Cyclopsetta querna & Lenguado dentón & Toothed flounder \\
Sphyraena ensis & Barracuda & Barracuda, Mexican barracuda \\
Cynoponticus coniceps & Anguila & Red pike conger \\
Pleurocodes planipes & Langostino & Pelagic red crab \\
Lolliguncula panamensis & Calamar torpedo & Panama brief squid \\
Anodonta luteola & Almeja de agua dulce & Freshwater Mussels \\
\hline
\end{tabular}

Nota: Fuente propia de la investigación.

Las muestras se obtuvieron en diferentes meses del año y fueron proporcionadas por pescadores artesanales y barcos camaroneros que operan en el Golfo de Nicoya. De cada especie de pescado se obtuvieron diversos ejemplares para alcanzar un mínimo de 12 individuos de cada una. Las muestras fueron colocadas en una hielera con hielo y trasladadas inmediatamente al laboratorio donde a cada ejemplar se le determinó la longitud y peso total, además se evisceraron, lavaron y filetearon para obtener la porción comestible equivalente a filete sin espinas. Treinta calamares e igual número de langostinos fueron transportados al laboratorio en hielera con hielo, en donde se midieron, pesaron y se les extrajo la porción comestible (manto y cefalotórax o cola, respectivamente).

Las almejas fueron obtenidas de estanques de tierra ubicados en la Estación Experimental Enrique Jiménez-Núñez, situada en Cañas, Guanacaste. 15 ejemplares fueron trasportados vivos hasta el laboratorio donde se determinó la longitud y el peso total. Luego el tejido blando de los individuos fue removido y drenado por 10min, y se registró su peso fresco.

Se utilizó un ictiometro y un vernier Fowler $( \pm 0,05 \mathrm{~mm}$ de precisión) para obtener las diferentes longitudes. El peso se registró utilizando una balanza electrónica AND GX-2000 ( $\pm 0,01 \mathrm{~g}$ de precisión).

Finalmente, el material fresco de cada especie fue molida y homogenizada para posteriormente obtener al azar $100 \mathrm{~g}$ para llevar a cabo los análisis químicos por triplicado (Castro-González et al., 2010).

\section{Análisis químico proximal}

A cada una de las muestras se le realizó el análisis químico proximal de los componentes de la carne, según la metodología de $\mathrm{AOAC}$ (1984): humedad por deshidratación en horno a $100^{\circ} \mathrm{C}$ por $24 \mathrm{~h}$; las cenizas se obtuvieron por calcinación lenta, incrementando la temperatura hasta $500^{\circ} \mathrm{C}$, donde se mantuvo por 12 horas (Crisp, 1971); la proteína cruda se obtuvo determinando el nitrógeno proteico por el método de Kjendhal y se convirtió a proteína multiplicando por el factor 6,25 (Crisp, 1971); el extracto etéreo fue obtenido usando un extractor Soxhlet (Lab-Line Instruments, Inc., ILL, USA) con éter de petróleo.

\section{Resultados y discusión}

En la Tabla 2 se presentan los resultados del análisis químico proximal de las seis especies de pescado y mariscos estudiadas. El agua fue el componente más abundante, con porcentajes entre 
UNICIENCIA Vol. 31, No. 1, pp. 23-28. Enero-junio, 2017.

$77,13 \%$ para la barracuda y $83,12 \%$ para el calamar torpedo. Estos valores son coincidentes con otros reportados para otras especies de pescado (Castro-González et al., 1998; Castro-González et al., 2007; Fonseca et al., 2013; Izquierdo et al., 2000; Izquierdo et al. 2001; Tawfik, 2009) y para el langostino (Torres, 2007). No se encontraron registros para el calamar y la almeja de agua dulce.

Tabla 2

Composición proximal (\%) de 6 especies de pescado y mariscos

\begin{tabular}{lcccc}
\hline Nombre & Humedad & Ceniza & Extracto etéreo & Proteínas \\
\hline Lenguado & $78,93 \pm 0,75$ & $1,31 \pm 0,75$ & $1,18 \pm 0,21$ & $19,86 \pm 0,73$ \\
Barracuda & $77,13 \pm 1,35$ & $1,21 \pm 0,08$ & $2,18 \pm 1,18$ & $19,52 \pm 0,69$ \\
Anguila & $78,50 \pm 1,12$ & $1,41 \pm 0,09$ & $1,99 \pm 0,21$ & $19,53 \pm 0,86$ \\
Langostino & $83,01 \pm 1,50$ & $1,51 \pm 0,44$ & $1,16 \pm 0,28$ & $13,52 \pm 1,15$ \\
Calamar torpedo & $83,12 \pm 1,66$ & $0,84 \pm 0,14$ & $1,46 \pm 0,25$ & $13,60 \pm 0,80$ \\
Almeja de agua dulce & $80,50 \pm 0,70$ & $1,65 \pm 1,15$ & $2,24 \pm 1,83$ & $10,73 \pm 1,07$ \\
\hline
\end{tabular}

Nota: Elaboración propia de la investigación.

Después de la humedad, el porcentaje de proteínas fue el de mayor importancia. Varío entre $10,73 \%$ para la almeja de agua dulce y 19,86\% para el lenguado. Las 3 especies de pescado estudiadas presentaron un promedio de 19,64\%. Estos valores son similares a los encontrados en otras carnes de alto contenido proteico, como la de vacuno, pollo y cerdo, que satisfacen los requerimientos mínimos diarios de la dieta del ser humano (Bourgeois y Le Roux, 1986; Stansby, 1963).

El contenido de extracto etéreo varió entre 1,16\% del calamar y 2,24\% de la almeja de agua dulce. Todas las especies estudiadas presentaron porcentajes de grasa inferiores al $3 \%$. De acuerdo con la clasificación propuesta por Varlik et al., (2004, citado por Özden et al., 2010), las especies estudiadas en este trabajo fueron consideradas como magras $(<4 \mathrm{~g} / 100 \mathrm{~g})$, lo cual puede ser más atractivo para quienes prefieren carnes blancas. Similares resultados han sido reportados por Özden et al., (2010) para Solea solea y Merlangius merlangus e Izquierdo et al., (2001) y Romero et al., (2000) para el robalo C. undecimalis.

En relación con el contenido de ceniza, todas las especies mostraron un bajo contenido de este elemento; el calamar fue la especie que presentó el menor porcentaje $(0,84 \%)$ y la almeja de agua dulce el mayor (1,65\%).

\section{Conclusiones}

Los resultados permiten concluir que las especies estudiadas son una buena alternativa para el consumo humano, por su bajo contenido en grasas y por el aporte proteico, principalmente en el caso de las especies de pescado, el langostino y el calamar. Autores como Fonseca et al. (2013), Luzia et al. (2003) y Tawfik, 2009 al analizar el contenido químico proximal de otras especies marinas de importancia comercial expresaron conclusiones similares a las nuestras.

En futuras investigaciones se recomienda continuar los estudios sobre el valor nutricional de otras especies de pescado y mariscos en Costa Rica, haciendo énfasis en el perfil de aminoácidos, ácidos grasos y minerales. 
ISSN Electrónico: 2215-3470

DOI: http://dx.doi.org/10.15359/ru.31-1.3
UNICIENCIA Vol. 31, No. 1, pp. 23-28. Enero-junio, 2017.

URL: www.revistas.una.ac.cr/uniciencia Email: revistauniciencia@una.cr

\section{Referencias}

AOAC. (1984). Official methods of analysis. Washington D.C., EE.UU.: Association of Official Analytical Chemists. Astorga, M., Rodríguez, E. \& Díaz, C. (2007). Comparison of minerals and trace element concentration in two mollusks from the Strait of Magellan (Chile). J. Food Comp. Annual, 20, 273-279. http:// dx.doi.org/10.1016/j.jfca.2006.06.007

Badillo-Zapata, D., G. Correa-Reyes, L., D’Abramo, J., Lazo, J., Toro-Vázquez \& M. Viana. (2010). Effect of replacing dietary fish oil with vegetable oils on the fatty acid composition of muscle tissue of juvenile California halibut (Paralichthys californicus). Cienc. Mar., 36, 121-133. http://dx.doi.org/10.7773/ cm.v36i2.1637

Báez, R. (1985). Evaluación de algunos aspectos de la biología del molusco Polymesoda inflata (Philipp, 1851) (Bivalvia: Corbiculidae), en el manglar de Pochote, Puntarenas, Costa Rica (Tesis de licenciatura). Universidad de Costa Rica, San José, Costa Rica.

Blanco, A. \& Montero, M. (1992). Composición químico-nutricional de la carne de cambute, Strombus galeatus (Mesogastropoda: Strombidae). Rev. Biol. Trop., 40, 89-93.

Bourgeois, C. y Le Roux, P. (1986). Proteinas animales. México D.F.: El Manual Moderno.

Cabrera, T., Cabrera, G., Rosas, J., Velásquez A. y Silva, M. (2005). Variación de lípidos y ácidos grasos en camarones marinos consumidos en Venezuela. ALAN 55, 194-200.

Castro-González, M., Silencio, J., Juárez, M., Montaño, S. \& Pérez-Gil, F. (1998). Composición química de la fauna de acompañamiento del camarón de Veracruz (Golfo de México). Rev. Biol. Trop., 46, 249-256.

Castro-González, M., Ojeda., V. A., Montaño, B.S. Ledesma, C.E. \& Pérez-Gil, R.F. (2007). Evaluación de los ácidos grasos n-3 de 18 especies de pescados marinos mexicanos como alimentos funcionales. ALAN 57, 85-93.

Castro-González, M. I., Miranda-Becerra, D. (2010). El pescado en la dieta del paciente renal: Relación fósforo: ácidos grasos n-3. Rev Invest Clin., 62(1), 44-53.

Chukwu, O. y Mohammed, I. (2009). Effects of Drying Methods on Proximate Compositions of Catfish (Clarias gariepinus). World J. Agric. Sci., 5, 114-116.

Cox, K. y Hartman, K. (2005). Nonlethal estimation of proximate composition in fish. Canadian Journal of Fisheries and Aquatic Sciences, 62(2), 269-275. http://dx.doi.org/10.1139/f04-180

Crisp, D. J. (1971). Energy flow measurements. En N. A. Holme y A. D. McIntyre (Eds.), Methods for the study of marine benthos (pp. 197-278). Blackwell, Oxford: IBP Handbook.

Cruz, R. A., Fonseca, C. y Chavarría, F. (2012). Comparación de la composición química proximal de la carne de Anadara tuberculosa y A. similis (Bivalvia: Arcidae) de Chomes, Puntarenas, Costa Rica. Rev. Mar. Cost., 4, 95-103

Cruz, R. A., y Villalobos, C. R. (1993). Monthly changes in tissue weight and biochemical composition of the mussel Mytella guyanensis (Bivalvia: Mytillidae) in Costa Rica. Rev. Biol. Trop., 41, 93-96.

FAO. (1991). Fish for Food and Development. Food and Agriculture Organization of the United Nations. Rome, Italy: Autor.

Fawole, O. O., Ogundiran, M. A., Ayandiran, T. A., y Olagunju, O. F. (2007). Proximate and mineral composition in some selected fresh water fishes in Nigeria. Internet Journal of Food Safety 9, 52-55.

Fonseca, C., Chavarría, F. y Mejía-Arana, F. (2013). Variación estacional de la composición proximal en tres especies de importancia comercial del Golfo de Nicoya, Puntarenas, Costa Rica. Rev. Biol. Trop., 61(1), 429-437.

Fonseca, C., Marín-Vindas, C., Chavarría-Solera, F., Cruz, R.A. y Toledo, P. (2011). Variación estacional de la composición proximal del mejillón Tagelus peruvianus (Bivalvia: Solecurtidae) del Golfo de Nicoya, Puntarenas, Costa Rica. Rev. Biol. Trop., 54, 1517-1523. 
Fuentes, A., I. Fernández-Segovia, I. Escrichey J. Serra.(2009). Comparison of physicochemical parameters and composition of mussels (Mytilus alloprovincialis Lmk.) from different Spanish origins. Food Chem., 112, 295-302. http://dx.doi.org/10.1016/j.foodchem.2008.05.064

Izquierdo, P., Torres, G., Barboza, Y., Márquez, S. y Allara, M. (2000). Análisis proximal, perfil de ácidos grasos, aminoácidos esenciales y contenido de minerales en doce especies de pescado de importancia comercial en Venezuela. ALAN 50, 187-194.

Izquierdo, P., Torres, G., Allara, M., Marquez, E., Barboza, Y., y Sanchez, E. (2001). Análisis proximal, contenido de aminoácidos esenciales y relación calcio/fosforo en algunas especies de pescado. FCV-LUZ, 2, 95-100.

Jiménez-Arce, G. (1993). Composición químico-nutricional en diferentes tallas y sexos del cambute Strombus gracilior (Mesogastropoda: Strombidae) de Playa Panamá, Costa Rica. Rev. Biol. Trop., 41, 345-349.

López, L., Durazo, E., Rodríguez-Gómez, A., True, C. y Viana, M. (2006). Composición proximal y perfil de ácidos grasos de juveniles silvestres y cultivados de Totoaba macdonaldi. Cienc. Mar., 32, 303-309.

Luzia, L.A., G.R. Sampaio, C.M. Castellucci \& E.A. Torres. (2003). The influence of season on the lipid profiles of five commercially important species of Brazilian fish. Food Chem, 83, 93-97.

Nazrul, I. y Razzaq, A. (2005). Seasonal variation of the proximate composition of freshwater gobi, Glossogius giuris (Hamilton) from the River Padma. Pak. J. Biol. Sci., 8, 532-536. http://dx.doi. org/10.3923/pjbs.2005.532.536

Nurnadia, A. A., Azrina, A., \& Amin, I. (2011). Proximate composition and energetic value of selected marine fish and shellfish from the West coast of Peninsular Malaysia. International Food Research Journal, 18(1), 137-148.

Özden, Ö. (2010a). Seasonal differences in the trace metal and macrominerals in shrimp (Parapenaus longirostris) from Marmara Sea. Environ. Monit. Assess, 162, 191-199. http://dx.doi.org/10.1007/s10661-009-0787-y

Özden, Ö. (2010b). Micro, macro mineral and proximate composition of Atlantic bonito and horse mackerel: a monthly differentiation. Int. J. Food Sci. Technol., 45, 578-586. http://dx.doi. org/10.1111/j.1365-2621.2009.02170.x

Özden, Ö., Erkan, N. y Ulusoy, . (2010). Determination of mineral composition in three commercial fish species (Solea solea, Mullus surmuletus and erlangius merlangus). Environ. Monit. Assess., 170, 353363. http://dx.doi.org/10.1007/s10661-009-1238-5

Perea, A., Gómez, E., Mayorga, Y., y Triana, C.Y. (2008). Caracterización nutricional de pescados de producción y consumo regional en Bucaramanga, Colombia. Archivos Latinoamericanos de Nutrición, 58, 91-97.

Romero, N., P. Robert, L. Mansson y R Pined. (2000). Composición de ácidos grasos y proximal de siete especies de pescado de Isla de Pascua. ALAN 50, 304-308.

Ruiz, E., Cabrera, J., Cruz, R. A. y Palacios, J. (1998). Crecimiento y ciclo reproductivo de Polymesoda radiata (Bivalvia: Corbiculidae) en Costa Rica. Rev. Biol. Trop., 46, 643-648.

Stansby, M. (1962). Analytical Methods, Industrial Fishery Technology. Londres, Inglaterra: Chapman y Hall.

Stansby, M. (1963). Industrial Fishery Technology. Londres, Inglaterra: Chapman y Hall.

Tawfik, M. S. (2009). Proximate composition and fatty acids profiles in most common available fish species in Saudi Martket. Asian J. Clin. Nutr., 1, 50-57. http://dx.doi.org/10.3923/ajcn.2009.50.57

Torres, E. (2007). Valoración de la calidad de un extracto desgrasado de langostilla (Pleuroncodes planipes), sometido a diferentes métodos de conservación, como aditivo en alimentos para juveniles del camarón Litopenaeus vannamei (Tesis de maestría). Centro de Investigaciones Biológicas del Norte. La Paz, Bolívia.

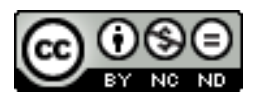

Composición proximal en algunas especies de pescado y mariscos disponibles en el pacífico costarricense (Cristian Fonseca-Rodríguez y otros) por Revista Uniciencia se encuentra bajo una Licencia CreativeCommons Atribución-NoComercial-SinDerivadas 3.0 Unported. 